\title{
Diffusive-Ballistic Transition in Random Polymers with Drift and Repulsive Long-Range Interactions
}

\author{
L.M. Ciolettia, 1, C.C.Y. Dorea ${ }^{\mathrm{a}}$ and S. Vasconcelos da Silva ${ }^{\mathrm{b}}$ \\ a Universidade de Brasilia, 70910-900 Brasilia-DF, Brazil \\ b Universidade Federal de Goias, 74001-970 Goiania-GO, Brazil
}

\begin{abstract}
In this note phase transition issues are addressed for random polymers on $\mathbb{Z}^{2}$ with long-range self-repulsive interactions. It is shown that, in the absence of drift and with power law interactions, the polymer exhibits transition from diffusive to a ballistic behavior. When non-null drifts are added and positive translation invariant interactions are considered, the polymer presents a ballistic behavior. Our results complement some previous studies on the matter and we also derive a Central Limit Theorem for the model.
\end{abstract}

MSC: 82B20, 82B41, 82B26.

Keywords: self-repelling random polymers; Ising model; long-range interactions; diffusiveballistic phase transition; CLT.

\section{Introduction}

Random polymers can be modelled as connected subsets of $\mathbb{Z}^{2}$. More precisely, a $N$-th step polymer $S$ is an element of $\mathbb{W}_{N}$ given by

$$
\mathbb{W}_{N}:=\left\{S=\left(S_{0}, S_{1}, \ldots, S_{N}\right): S_{i} \in \mathbb{Z}^{2}, S_{0}=0 \text { and }\left\|S_{i+1}-S_{i}\right\|=1\right\}
$$

being $\|\cdot\|$ the $\ell^{1}$ norm. Under Gibbs measure setting at inverse temperature $\beta>0$ and Hamiltonian $\mathbb{H}_{N}$ we can write the probability

$$
\mathbb{P}_{N}^{\beta, h}(S)=\frac{\exp \left[-\beta \mathbb{H}_{N}(S)\right]}{\mathbb{Z}_{N}^{\beta}(h)}, \quad \mathbb{H}_{N}(S)=-\sum_{1 \leq i<j \leq N} V_{i j}\left\langle X_{i}, X_{j}\right\rangle+\left\langle h, S_{N}\right\rangle,
$$

where $X_{i}=S_{i}-S_{i-1}$ stands for the $i$-th random step, $V_{i j}$ are the prescribed interactions, $\langle\cdot, \cdot\rangle$ denotes the usual inner product, $h \in \mathbb{R}^{2}$ is the fixed drift vector and $\mathbb{Z}_{N}^{\beta}(h)$ is the partition function.

\footnotetext{
${ }^{1}$ Corresponding author : leandro.mat@gmail.com

Co-authors : changdorea@unb.br ; simone@ufg.br

Research partially supported by CNPq and FEMAT.
} 
Caracciolo et al. ([3], 1993) introduced a self-repelling random polymer model with Hamiltonian on $\mathbb{W}_{N}$ given by $\bar{H}_{N}(S)=g_{0} \sum_{0 \leq i<j \leq N} V_{i j} \delta_{S_{i}, S_{j}}$, where $g_{0}>$ 0 and the interactions $V_{i j}=|i-j|^{-\alpha}$. Their model interpolates between the lattice Edwards model $(\alpha=0)$ and ordinary SRW $(\alpha=\infty)$. Moreover, it was conjectured that for dimension $1 \leq d \leq 4$ there exists a strictly positive exponent $\gamma=\gamma(d, \alpha)$ such that the mean square end-to-end distance satisfies the asymptotics

$$
\mathbb{E}_{\bar{P}_{N}}\left[\left\|S_{N}\right\|^{2}\right]=\sum_{S \in \mathbb{W}_{N}}\left\|S_{N}\right\|^{2} \bar{P}_{N}(S) \sim c N^{\gamma}
$$

where the Gibbs measure $\bar{P}_{N}$ is given by the Hamiltonian $\bar{H}_{N}$. In [10], the Hamiltonian $\tilde{H}_{N}(S)=-\sum_{0 \leq i<j \leq N}|i-j|^{-\alpha}\left\|S_{i}-S_{j}\right\|^{2}$, where $3<\alpha \leq 4$, was considered. They proved the existence of positive constants $\beta_{1}$ and $\beta_{2}$ that led to phase transition from diffusive regime $\left(\beta<\beta_{1}\right)$ to a ballistic one $\left(\beta>\beta_{2}\right)$. However, it was left unknown what undergoes when $\beta \in\left[\beta_{1}, \beta_{2}\right]$. As usual, the different diffusive regimes are classified according to the asymptotic behavior of the mean square displacement and for our model (1) it reduces in determining $\gamma>0$ for which the following limit exists, is positive and finite

$$
\lim _{N \rightarrow \infty} \frac{1}{N^{\gamma}} \mathbb{E}_{\mathbb{P}_{N}^{\beta, h}}\left[\left\|S_{N}\right\|^{2}\right]=\lim _{N \rightarrow \infty} \frac{1}{N^{\gamma}} \sum_{S \in \mathbb{W}_{N}}\left\|S_{N}\right\|^{2} \mathbb{P}_{N}^{\beta, h}(S) .
$$

We say that the polymer model is diffusive if $\gamma=1$, superdiffusive if $1<\gamma<2$ and ballistic if $\gamma=2$.

Our main motivation is to build a self-repelling random polymer model for which we can derive a genuine diffusive-ballistic phase transition, i.e. the existence of a unique positive constant $\beta_{c}$ separating the model into two regimes. In this note, assuming zero drift and $V_{i j}=|i-j|^{-\alpha}$ with $1<\alpha \leq 2$, we prove (Theorem 3) that there exists a unique positive number $\beta_{c}$ (the critical temperature of a related one dimensional Ising model) such that the model is diffusive for $\beta<\beta_{c}$ and ballistic for $\beta>\beta_{c}$. On the other hand, considering non-null drift and positive, translation invariant and regular interactions, we conclude from Theorem 2 that for all $\beta \in(0, \infty)$ the model is ballistic.

The Lemma 1 is an essential tool in this work. Its proof is similar in spirit to the one introduced for the Potts model by M. Suzuki [13] in 1967. It consists in decoupling the steps of the polymer as two independent Ising random variables. The background idea is the same applied when looking at SRW in lattice $\mathbb{Z}^{2}$ as two independents SRW's on $\mathbb{Z}$.

In 1983 Newman [9] proved a CLT for block random variables satisfying the FKG inequalities under finite susceptibility hypothesis. In Section 3 we investigate the validity of the CLT for our model. Here assuming non zero drifts and consequently infinite susceptibility, we prove (Theorem 4) a CLT for the projections of suitably normalized displacements. This is obtained by using both the Lee-Yang circle theorem and the $C^{2}$-regularity condition from $\mathrm{Wu}$ Liming [8]. 


\section{Mean Square Displacement and Phase Tran- sition}

For the volume $\Lambda_{N}=\{1,2, \ldots, N\}$ consider the one dimensional Ising model with free boundary conditions defined by the Hamiltonian

$$
H_{\Lambda_{N}}(\sigma)=-\sum_{1 \leq i<j \leq N} V_{i j} \sigma_{i} \sigma_{j}-\sum_{i=1}^{N} h \sigma_{i}
$$

where $\sigma=\left(\sigma_{1}, \ldots, \sigma_{N}\right) \in\{-1,1\}^{N}:=\Sigma_{N}, V_{i j} \in \mathbb{R}$ are the coupling constants and $h \in \mathbb{R}$ is an external field. To simplify notation for a given a real-valued function $f: \Sigma_{N} \rightarrow \mathbb{R}$ write

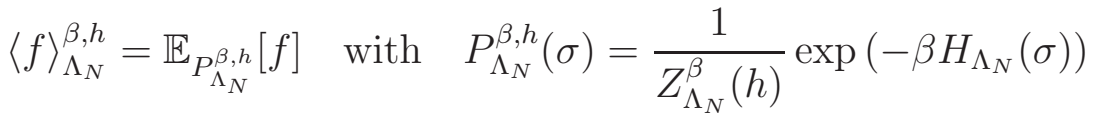

where $Z_{\Lambda_{N}}^{\beta}(h)$ is the partition function.

Lemma 1. For $e_{1}=(1,0)$ and $e_{2}=(0,1)$ define $h_{1}=\left\langle h, e_{1}-e_{2}\right\rangle$ and $h_{2}=$ $\left\langle h, e_{1}+e_{2}\right\rangle$. Then

$$
\mathbb{E}_{\mathbb{P}_{N}^{\beta, h}}\left[\left\|S_{N}\right\|^{2}\right]=\frac{1}{2} \sum_{i, j=1}^{N}\left[\left\langle\sigma_{i} \sigma_{j}\right\rangle_{\Lambda_{N}}^{\frac{\beta}{2}, h_{1}}+\left\langle\sigma_{i} \sigma_{j}\right\rangle_{\Lambda_{N}}^{\frac{\beta}{2}, h_{2}}\right]
$$

Proof. The proof follows closely the ideas from [2, 10]. Let $T: \mathbb{R}^{2} \rightarrow \mathbb{R}^{2}$ be the rotation $T X_{i}=\left(\sigma_{i} e_{1}+\tilde{\sigma}_{i} e_{2}\right) / \sqrt{2}$, with $\sigma_{i}, \tilde{\sigma}_{i} \in\{-1,1\}$. A simple computation shows that

$$
\mathbb{P}_{N}^{\beta, h}(S)=P_{\Lambda_{N}}^{\frac{\beta}{2}, h_{1}}(\sigma) P_{\Lambda_{N}}^{\frac{\beta}{2}, h_{2}}(\tilde{\sigma}) \quad \text { and } \quad\left\|S_{N}\right\|^{2}=\frac{1}{2} \sum_{i, j=1}^{N}\left(\sigma_{i} \sigma_{j}+\tilde{\sigma}_{i} \tilde{\sigma}_{j}\right) .
$$

Theorem 2. Suppose that $V_{i j}$ is positive and tranlation invariant, i.e. $V_{i j}=$ $V(|i-j|)>0$ for all $i \neq j$. If $h \in \mathbb{R}^{2}$ is such that $h_{1}$ and $h_{2}$ satisfy $h_{1} h_{2}>0$ and $\beta>0$, then for some constant $C(\beta, h)>0$ we have

$$
C(\beta, h) \leq \frac{\mathbb{E}_{\mathbb{P}_{N}^{\beta, h}}\left[\left\|S_{N}\right\|^{2}\right]}{N^{2}}
$$

Proof. Let $k \in \mathbb{R}$. Since $V_{i j}>0$ for $i \neq j$ we get from the second Griffiths inequality,

$$
\left\langle\sigma_{i}\right\rangle_{\Lambda_{N}}^{\beta, k, n n}\left\langle\sigma_{j}\right\rangle_{\Lambda_{N}}^{\beta, k, n n} \leq\left\langle\sigma_{i} \sigma_{j}\right\rangle_{\Lambda_{N}}^{\beta, k, n n} \leq\left\langle\sigma_{i} \sigma_{j}\right\rangle_{\Lambda_{N}}^{\beta, k}
$$


where the left hand side expected values are taken with respect to the Gibbs measure of the nearest neighbours Ising model on $\Lambda_{N}$ with free boundary conditions and Hamiltonian given by $H_{\Lambda_{N}}(\sigma)=-\sum_{n=1}^{N-1} V(1) \sigma_{n} \sigma_{n+1}-k \sum_{n=1}^{N} \sigma_{n}$. Using monotonicity with respect to the volume and classical transfer matrix computation (see [5, p. 107]) we have for any $i \in \Lambda_{N}$

$$
\left\langle\sigma_{i}\right\rangle_{\Lambda_{N}}^{\beta, k, n n} \underset{N \rightarrow \infty}{\longrightarrow} \frac{\sinh \beta k}{\sqrt{\sinh ^{2}(\beta k)+e^{-4 \beta V(1)}}} .
$$

Theorem 3. Let $1<\alpha \leq 2, h=0$ and $V_{i j}=|i-j|^{-\alpha}$ for $i \neq j$. Then there exist a constant $\beta_{c} \in(0, \infty)$ and positive numbers $m_{*}(\beta)$ and $K(\beta)$ such that

$$
\frac{1}{2} m_{*}^{2}(\beta) \leq \frac{1}{N^{2}} \mathbb{E}_{\mathbb{P}_{N}^{2 \beta, 0}}\left[\left\|S_{N}\right\|^{2}\right] \leq 1, \quad \text { if } \quad \beta>\beta_{c}
$$

and

$$
1 \leq \frac{1}{N} \mathbb{E}_{\mathbb{P}_{N}^{2 \beta, 0}}\left[\left\|S_{N}\right\|^{2}\right] \leq K(\beta), \quad \text { if } \quad 0<\beta<\beta_{c} .
$$

Proof. For $1<\alpha \leq 2$ the existence of a critical $\beta_{c} \in(0, \infty)$ for the long range Ising model with coupling $V_{i j}$ is shown in [4, 6]. In this case, we have spontaneous magnetization $m_{*}(\beta)>0$ for all $\beta>\beta_{c}$ and the two-point function with free boundary condition satisfies (cf. [7]),

$$
\left\langle\sigma_{i} \sigma_{j}\right\rangle^{\beta, 0}=\frac{1}{2}\left[\left\langle\sigma_{i} \sigma_{j}\right\rangle^{\beta, 0,+}+\left\langle\sigma_{i} \sigma_{j}\right\rangle^{\beta, 0,-}\right] \geq m_{*}^{2}(\beta) \geq m_{*}^{2}\left(\beta_{c}\right) .
$$

Using the same type of arguments as in Theorem 2 we have for large $N$

$$
\mathbb{E}_{\mathbb{P}_{N}^{2 \beta, 0}}\left[\left\|S_{N}\right\|^{2}\right]=\sum_{i, j=1}^{N}\left\langle\sigma_{i} \sigma_{j}\right\rangle_{\Lambda_{N}}^{\beta, 0} \geq \frac{1}{2} \sum_{i, j=1}^{N}\left\langle\sigma_{i} \sigma_{j}\right\rangle^{\beta, 0} \geq \frac{1}{2} m_{*}^{2}(\beta) N^{2} .
$$

To prove (44) one needs lower and upper bounds for $\left\langle\sigma_{i} \sigma_{j}\right\rangle_{\Lambda_{N}}^{\beta, 0}$. From the monotonicity with respect to the volume and [1], if $\beta<\beta_{c}$ there are constants $0<C(\beta) \leq C^{\prime}(\beta)<\infty$ such that $\left\langle\sigma_{i} \sigma_{j}\right\rangle_{\Lambda_{N}}^{\beta, 0}$ with free boundary condition satisfies

$$
\frac{C(\beta)}{|i-j|^{\alpha}} \leq\left\langle\sigma_{i} \sigma_{j}\right\rangle_{\Lambda_{N}}^{\beta, 0} \leq\left\langle\sigma_{i} \sigma_{j}\right\rangle_{\mathbb{N}}^{\beta, 0} \leq \frac{C^{\prime}(\beta)}{|i-j|^{\alpha}},
$$

where $C(\beta) \equiv\left(\beta \tanh \beta_{c}\right) / \beta_{c}$. The uniformity of the lower bound is a simple application of FKG inequality. Using Lemma 1 we have

$$
N+\sum_{1 \leq i<j \leq N} \frac{2 C(\beta)}{|i-j|^{\alpha}} \leq \mathbb{E}_{\mathbb{P}_{N}^{2 \beta, 0}}\left[\left\|S_{N}\right\|^{2}\right] \leq N+\sum_{1 \leq i<j \leq N} \frac{2 C^{\prime}(\beta)}{|i-j|^{\alpha}} .
$$

Inequality (41) follows by observing that $\sum_{1 \leq i<j \leq N} \frac{1}{|i-j|^{\alpha}}=\mathcal{O}(N)$. 


\section{Central Limit Theorem}

To derive a CLT for (1) we make use of Theorem 1.2 and Theorem 3.1 from [8]. It is required that a $C^{2}$-regularity condition to be satisfied. We say that a sequence of probability measures $\left\{\mu_{N}\right\}$ satisfies the $C^{2}$-regularity condition if for $Y_{N}$ with probability measure $\mu_{N}$ the following limit exists

$$
\Psi(t)=\lim _{N \rightarrow \infty} \Psi_{N}(t)=\lim _{N \rightarrow \infty} \frac{1}{N} \ln \mathbb{E}_{\mu_{N}}\left[\exp \left(t N Y_{N}\right)\right] .
$$

Moreover, for some neighborhood $[-\delta, \delta]$ of zero we have $\Psi(\cdot)<\infty$ and

$$
\Psi_{N}^{\prime \prime}(t) \underset{N \rightarrow \infty}{\longrightarrow} \Psi^{\prime \prime}(t) \text { uniformly on }[-\delta, \delta]
$$

Under these hypotheses $Y_{N}$ is asymptotically Gaussian.

For our polymer model take $v \in \mathbb{R}^{2}$ fixed and consider the empirical field projection

$$
L_{N}=\frac{1}{N}\left\langle S_{N}, v\right\rangle=\frac{1}{N} \sum_{j=1}^{N}\left\langle X_{j}, v\right\rangle .
$$

Set $\mu_{N}=\mathbb{P}_{N}^{\beta, h}$ and define the pressure functional by

$$
\Psi^{\beta, h, v}(t)=\lim _{N \rightarrow \infty} \Psi_{N}^{\beta, h, v}(t)=\lim _{N \rightarrow \infty} \frac{1}{N} \ln \mathbb{E}_{\mathbb{P}_{N}^{\beta, h}}\left[\exp \left(\beta t N L_{N}\right)\right]
$$

Theorem 4. Assume that the interactions are translation invariant and summable, that is, $V_{i j}=V(|i-j|)>0$ and $\sum_{i \in \mathbb{N}} V(i)<\infty$. For $h \in \mathbb{R}^{2}$ with $h_{1} h_{2} \neq 0$ and any fixed $v \in \mathbb{R}^{2}$ we have

$$
\frac{1}{\sqrt{N}}\left[\beta\left\langle S_{N}, v\right\rangle-N \mathbb{E}_{\mathbb{P}_{N}^{\beta, h}}\left[\beta\left\langle S_{N}, v\right\rangle\right]\right] \stackrel{\mathscr{P}}{\rightarrow} N\left(0, \frac{\partial^{2}}{\partial t^{2}} \Psi^{\beta, h, v}(0)\right)
$$

where " $\rightarrow$ " stands for convergence in distribution.

Proof. Under the hypotheses, the existence of the limit $\Psi^{\beta, h, v}(\cdot)$ is proved in [12]. To complete the $C^{2}$-regularity verification take complex number $z \in \mathbb{C}$ and express $\Psi_{N}^{\beta, h, v}(z)$ in terms of partition functions of one-dimensional Ising model. As in Lemma 1 write $\mathbb{Z}_{N}^{\beta}(h)=Z_{\Lambda_{N}}^{\beta / 2}\left(h_{1}\right) Z_{\Lambda_{N}}^{\beta / 2}\left(h_{2}\right)$. Using the principalvalue logarithm identities

$$
\begin{gathered}
\ln (z w)=\ln z+\ln w+2 \pi i \mathcal{K}(\ln z+\ln w) \\
\ln (z / w)=\ln z-\ln w+2 \pi i \mathcal{K}(\ln z-\ln w)
\end{gathered}
$$


where $\mathcal{K}(x+i y)=-\sum_{n \geq-1} n I((2 n-1) \pi<y \leq(2 n+1) \pi)$ with $I(\cdot)$ being the indicator function, we have for $v_{1}=\left\langle v, e_{1}-e_{2}\right\rangle$ and $v_{2}=\left\langle v, e_{1}+e_{2}\right\rangle$

$$
\begin{aligned}
\Psi_{N}^{\beta, h, v}(z)= & \frac{1}{N}\left[\ln \mathbb{Z}_{N}^{\beta}(h+z v)-\ln \mathbb{Z}_{N}^{\beta}(h)+2 \pi i \mathcal{K}\left(\ln \mathbb{Z}_{N}^{\beta}(h+z v)-\ln \mathbb{Z}_{N}^{\beta}(h)\right)\right] \\
= & \frac{1}{N} \ln Z_{\Lambda_{N} / 2}^{\beta / 2}\left(h_{1}+z v_{1}\right)+\frac{1}{N} \ln Z_{\Lambda_{N}}^{\beta / 2}\left(h_{2}+z v_{2}\right) \\
& +\frac{2 \pi i}{N} \mathcal{K}\left(\ln Z_{\Lambda_{N}}^{\beta / 2}\left(h_{1}+z v_{1}\right)+\ln Z_{\Lambda_{N}}^{\beta / 2}\left(h_{2}+z v_{2}\right)\right) \\
& -\frac{1}{N} \ln Z_{\Lambda_{N}}^{\beta / 2}\left(h_{1}\right)-\frac{1}{N} \ln Z_{\Lambda_{N}}^{\beta / 2}\left(h_{2}\right)+\frac{2 \pi i}{N} \mathcal{K}\left(\ln \mathbb{Z}_{N}^{\beta}(h+z v)\right) .
\end{aligned}
$$

By assuming that $\operatorname{Re}\left(h_{i}+z v_{i}\right) \neq 0$ and $h_{1} h_{2} \neq 0$ it follows from Lee-Yang Theorem's and standard arguments from [11, p. 111] that

$$
\Psi_{N}^{\beta, h, v}(z) \rightarrow \Psi^{\beta, h, v}(z) \text {, locally uniformly in } z \text {. }
$$

Also, it follows that the derivatives of $\Psi_{N}^{\beta, h, v}(z)$ converge uniformly on the compact subsets of $\mathbb{C}$. Hence the $C^{2}$-regularity condition is satisfied. Since

$$
\left.\frac{\partial^{2}}{\partial t^{2}} \Psi_{N}^{\beta, h, v}(t)\right|_{t=0}=\frac{1}{N} \mathbb{E}_{\mathbb{P}_{N}^{\beta, h}}\left[\beta\left\langle S_{N}, v\right\rangle-N \mathbb{E}_{\mathbb{P}_{N}^{\beta, h}}\left[\beta\left\langle S_{N}, v\right\rangle\right]\right]^{2} \rightarrow \frac{\partial^{2}}{\partial t^{2}} \Psi^{\beta, h, v}(0)
$$

we conclude the proof using Theorem 3.1 from [8].

Remark 1. We emphasize that in the above theorem we proved more than $C^{2}$ regularity condition. In fact, we proved that the pressure is analytic. Another way to obtain the $C^{2}$-regularity condition for our polymer model is to apply both FKG and GHS inequalities, see [8, p. 426].

\section{Concluding Remarks}

The random polymer model considered here interpolates between the SRW (infinite temperature) and a deterministic straight line (zero temperature). At very high temperatures this random polymer should be recurrent and transience would occur at very low temperatures, so we expect a recurrence-transience phase transition. It would be interesting to prove the existence of such phase transition and also to determine the critical temperature that separates these two regimes.

Acknowledgments. We are grateful to L.R. Fontes for his many valuable comments and careful reading of this manuscript. 


\section{References}

[1] M. Aizenman, J. T. Chayes, L. Chayes, C. M. Newman. Discontinuity of the magnetization in one-dimensional $1 /|x-y|^{2}$ Ising and Potts models, J. Statist. Phys. 50, 1-40 (1988)

[2] P. Buttà, A. Procacci, B. Scoppola. Kac polymers, J. Statist. Phys. 119, no. 3-4, 643-658 (2005)

[3] S. Caracciolo, G. Parisi, A. Pelissetto. Random Walks with short-range interaction and mean field behaviour, J. Statist. Phys., 77, no. 3-4, 519-543 (1994)

[4] F. J. Dyson. Existence of a phase-transition in a one-dimensional Ising ferromagnet. Comm. Math. Phys. 12, no. 2, 91-107 (1969)

[5] Richard S. Ellis. Entropy, Large Deviation and Statistical Mechanics. Springer. (2005)

[6] J. Fröhlich, T. Spencer. The phase transition in the one-dimensional Ising model with $1 / r^{2}$ interaction energy. Comm. Math. Phys. 84, no. 1, 87-101 (1982)

[7] J. L. Lebowitz. Coexistence of Phases in Ising Ferromagnets, J. Statist. Phys., 16, no. 6, 463-476 (1977)

[8] Liming, Wu. Moderate Deviations of Dependent Random Variables Related to CLT, Ann. Probab., 23, no. 1, 420-445 (1995).

[9] C. M. Newman. A General Central Limit Theorem for FKG Systems, Comm. Math. Phys. 91, 75-80 (1983)

[10] A. Procacci, R. Sanchis and B. Scoppola. Diffusive-Ballistic Transition in Random Walks with Long-Range Self-Repulsion, Lett. Math. Phys. 83, 181187 (2008).

[11] D. Ruelle. Statistical Mechanics - Rigorous Results, World Scientific, Imperial College Press. (2007).

[12] D. Ruelle. Thermodynamic Formalism, Addison-Wesley, Reading, MA. (1978).

[13] M. Suzuki. Solution of Potts Model for Phase Transition. Prog. Theor. Phys. 37 (1967) 